\title{
Stress cardiomyopathy in hip fracture. Differential diagnosis
}

\author{
Carlo Rostagno $^{1,2 *}$, Alessandra Cammilli ${ }^{2}$, Annalaura Di Cristo ${ }^{2}$, Gian Luca Polidori ${ }^{2}$, Claudia Ranalli ${ }^{2}$, Alessandro Cartei ${ }^{2}$ and Roberto \\ Buzzi $^{3}$ \\ ${ }^{1}$ Dipartimento Medicina Sperimentale e Clinica, Università di Firenze, Italy \\ ${ }^{2} \mathrm{SOD}$ medicina interna epost chirurgicaAOU Careggi, Italy \\ ${ }^{3} \mathrm{SOD}$ traumatologia ed Ortopedia AOU Careggi, Italy
}

Several studies suggest that early treatment of hip fracture is associated with a lower 30 days and long term mortality. Nevertheless patients with preoperative myocardial infarction hip surgery is associated with an high 30 day mortality (30-40\%) [1]. Among 1205 patients referred to Trauma Centre of AOU Careggi Florence October 12011 - September 30 2013, 5 (four women) out of 21 patients with preoperative significant troponin increase showed ST elevation at ECG. In 2 ECG changes were present at hospital admission while in 3 developed with in the first 24 hours of hospitalization. Average age was 86.2 years (range $78-92$ ). Troponin $\mathrm{T}$ rose in allpatients with a mean peak value of $3.16 \mathrm{ng} / \mathrm{ml}$ (range 0.52 to 5.53 ). The 92 yearmale patient sustainedhip fracture after accidental fall. At admission he complained chest pain and ECG showed anterior ST elevation. Severe left ventricular dysfunction was found at echocardiogram (LVEF 38\%). Due to severe cognitive and functional impairment a conservative treatment was suggested. He was discharged at home after clinical stabilization and died two months later due to heart failure. Among the other four patients none had history of coronary artery disease. All were treated since a long time for arterial hypertension. One suffered from moderate aortic stenosis, another was in treatment with oncocarbide for thrombocytosis. Two patients complained chest pain within 24 hours from hospital admission with ST elevation at ECG. Echocardiography showed apical akinesia with decreased left ventricular function. The third patient developed pulmonary edema while waiting for induction of anesthesia, ECG showed ST elevation and echocardiogram severe abnormality of apex wall motion. The last one developed two episodes of supraventricular arrhythmia, the first resolved with adenosine i.v., the second withvagal stimulation. She did not complain chest pain. Her baseline electrocardiogram showed sinus rhythm, normal AV conduction, and incomplete left bundle branch. During the SVT and after its resolution in leads V2-V4 ST segment elevation was appreciable. The echocardiogram showed severe apical hypokinesia and preserved all motion of basal segments. Left ventricular ejection fraction was $30 \%$.

Clinical, electrocardio graphic and echocardio graphic findings in these four patients fulfilled Mayo clinic criteria [2] for stress induced cardiomyopathy thus we decided to perform coronary angiography to confirm diagnostic hypothesis before hip surgery. No significant coronary lesions were demonstrated in all four patients. $\beta$-blockers and aspirin were added to treatment. Wall motion abnormalities improved significantly in all patients within 2-4 days after first examination.
Hip surgery was performed under general anaesthesia between day 4 and 11 after symptoms onset, (two patients were treated with synthesis of fracture, the other with hip prosthesis). The postoperative period was uneventful and patients were discharged to rehabilitation services. At one-year follow-up they were alive, three had recovered fully functional capacity.

Clinical presentation of stress cardiomyopathy may be undistinguishable from STEMI $[3,4]$. The syndrome has a large female prevalence in the seventh and eighth decade of life. At present only a few cases has been reported in trauma patients and only one in a patient with hipfracture [5]. Preoperative diagnosis has particular relevancein patients who need urgent non cardiac surgery. In fact in stress cardiomyoptahy the transient nature of segmental wall motion abnormalities and the absence of significant coronary stenosis are associated with a lowersurgical risk in comparison to patients with acute myocardial infarction. In elderly females with hip fracture ST segment elevation associated with apical "ballooning" pattern at echocardiographyshould raise the suspect of stress cardiomyopathy andcoronary angiography is suggested to confirm the diagnosis and schedule orthopedic surgery.

\section{Acknowledgement}

The paper is part of a project financed by a Grant from Italian Health Ministry and Regione Toscana (RF-2010-2316600).

\section{References}

1. Thiagarajah S, Fenton A, Sivardeen Z, Stanley D (2011) The management and mortality of patients undergoing hip fracture surgery following recent acute myocardial infarction Acta Orthop Belg 77: 626-631. [Crossref]

2. Bybee KA, Kara T, Prasad A, Barsness GW, Wright RS, et al. (2004) Systematic review: transient left ventricular apical ballooning: a syndrome that mimics ST-segment elevation myocardial infarction. Ann Intern Med 14: 858-865. [Crossref]

3. Prasad A, Lerman A, Rihal CS (2008) Apical ballooning syndrome (Tako-Tsubo or stress cardiomyopathy): a mimic of acute myocardial infarction. Am Heart J 155: 408417. [Crossref]

Correspondence to: Carlo Rostagno, Dipartimento Medicina Sperimentale e Clinica, Università di Firenze,Viale Morgagni 85 , 50134 - Firenze, Italy, Tel: +390557948545, Fax: +390557948547, E-mail: carlo.rostagno@unifi.it

Received: June 02, 2017; Accepted: June 24, 2017; Published: June 26, 2017 
4. Sharkey SW, Lesser JR, Zenovich AG, Maron MS, Lindberg J, et al. (2005) Acute and reversible cardiomyopathy provoked by stress in women from the United States. Circulation 111: 472-479. [Crossref]
5. Joe BH, Hwang HJ, Park CB, Jin ES, Sohn IS, et al. (2013) Takotsubo cardiomyopathy recurrence with left ventricular apical ballooning following isolated right ventricular involvement: A case report. Exp Ther Med 6: 260-262.[Crossref]

Copyright: (C2017 Rostagno C. This is an open-access article distributed under the terms of the Creative Commons Attribution License, which permits unrestricted use, distribution, and reproduction in any medium, provided the original author and source are credited. 